\title{
BMJ Global Health Impact of the COVID-19 pandemic and response on the utilisation of health services in public facilities during the first wave in Kinshasa, the Democratic Republic of the Congo
}

To cite: Hategeka C, Carter SE, Chenge FM, et al. Impact of the COVID-19 pandemic and response on the utilisation of health services in public facilities during the first wave in Kinshasa, the Democratic Republic of the Congo. BMJ Global Health 2021;6:e005955. doi:10.1136/ bmjgh-2021-005955

Handling editor Sanni Yaya

- Additional supplemental material is published online only. To view, please visit the journal online (http://dx.doi.org/10. 1136/bmjgh-2021-005955)

The findings of this study have been previously presented at the 2020 DHIS2 Annual Conference and the 2021 CUGH Annual Global Health Conference.

Received 8 April 2021 Accepted 24 June 2021

Check for updates

(C) Author(s) (or their employer(s)) 2021. Re-use permitted under CC BY-NC. No commercial re-use. See rights and permissions. Published by BMJ.

For numbered affiliations see end of article.

Correspondence to Dr Karen Ann Grépin; kgrepin@hku.hk

\section{ABSTRACT}

Introduction Health service use among the public can decline during outbreaks and had been predicted among low and middle-income countries during the COVID-19 pandemic. In March 2020, the government of the Democratic Republic of the Congo (DRC) started implementing public health measures across Kinshasa, including strict lockdown measures in the Gombe health zone.

Methods Using monthly time series data from the DRC Health Management Information System (January 2018 to December 2020) and interrupted time series with mixed effects segmented Poisson regression models, we evaluated the impact of the pandemic on the use of essential health services (outpatient visits, maternal health, vaccinations, visits for common infectious diseases and non-communicable diseases) during the first wave of the pandemic in Kinshasa. Analyses were stratified by age, sex, health facility and lockdown policy (ie, Gombe vs other health zones).

Results Health service use dropped rapidly following the start of the pandemic and ranged from $16 \%$ for visits for hypertension to $39 \%$ for visits for diabetes. However, reductions were highly concentrated in Gombe ( $81 \%$ decline in outpatient visits) relative to other health zones. When the lockdown was lifted, total visits and visits for infectious diseases and noncommunicable diseases increased approximately twofold. Hospitals were more affected than health centres. Overall, the use of maternal health services and vaccinations was not significantly affected.

Conclusion The COVID-19 pandemic resulted in important reductions in health service utilisation in Kinshasa, particularly Gombe. Lifting of lockdown led to a rebound in the level of health service use but it remained lower than prepandemic levels.

\section{INTRODUCTION}

At the outset of the COVID-19 pandemic, it was predicted that sub-Saharan Africa (SSA) was vulnerable to record large numbers of cases and deaths. ${ }^{1}$ To date, however, the

\section{Key questions}

What is already known?

- Substantial declines in the use of health services among the general population have been well documented during previous outbreaks of infectious diseases.

- Modelled studies predicted substantial increases in morbidity and mortality in many low and middleincome countries (LMICs) mainly due to expected declines in the use of health services among the general population.

- Only a small number of studies have so far evaluated the impact of the COVID-19 pandemic on the use of health services in LMICs and none have also evaluated both the implementation and lifting of lockdown measures.

What are the new findings?

- This study found that overall use of health services declined in Kinshasa but was most pronounced in the Gombe health zone which was subject to strict lockdown measures

- Some health services were more affected than others, most notably visits and tests for malaria and visits for new diagnoses of non-communicable diseases.

- Maternal and child health services were relatively unaffected.

- When the lockdown measures were lifted, health service utilisation rebounded but remained at levels lower than those observed prepandemic.

region has been relatively less affectedthrough 22 March 2021, it accounted for only approximately $2.5 \%$ of globally confirmed cases and a lower proportion of deaths. ${ }^{2}$ It is unclear, however, to what extent these levels may be explained by lower testing rates, less severe clinical presentation or other factors. 


\section{Key questions}

What do the new findings imply?

- The COVID-19 pandemic has likely had important effects on the use of health services among the general population throughout LMICs.

- However, evidence from Kinshasa suggests the effects may not be as widespread as previously predicted.

- The impact of strict social distancing measures on COVID-19 outcomes needs to be weighed off against the potential populationlevel health effects of these policies in various international contexts.

Studies have shown that outbreaks, as well as governmental response to outbreaks, can lead to important unintentional secondary health effects-or 'collateral damage'-mainly the result of reduced utilisation of health services for other conditions. For example, a systematic review of the West African Ebola virus disease (EVD) outbreak found an $18 \%$ decline in the overall use of health services. ${ }^{3}$ Some health services were more severely affected: reductions of $80 \%$ in facility deliveries, $40 \%$ reductions in malaria admissions and important reductions in immunisations were all documented. ${ }^{4}$ Modelling studies have suggested that mortality from non-Ebola conditions was potentially as large as the direct effects of EVD. ${ }^{5-8}$ Many factors likely led to decreased use of health services, including interruptions in treatment protocols, ${ }^{9-11}$ health worker mortality, ${ }^{12}$ as well as fear and a lack of trust in the health system. ${ }^{13} 14$ Persistent reductions in health service utilisation were also observed in the aftermath of the West African EVD outbreak. ${ }^{15-17}$

There are concerns that such patterns will be repeated during COVID-19, especially in SSA where sizeable increases in health service utilisation over the past few decades could be erased. Early modelled studies predicted that the pandemic could exert a devastating toll on health service utilisation and mortality. ${ }^{1819}$ Early predictions of the potential impact of health service disruptions on the burden of malaria in SSA suggested that under certain scenarios, malaria deaths could double in 2020 relative to $2018 .^{20}{ }^{21}$ Another study estimated that drops in the coverage of maternal and child health interventions could lead to substantial additional deaths in low and middle-income countries (LMICs). ${ }^{18}$ While modelled studies provide useful insights, their ability to accurately predict outcomes depends on the data available, the models used and the parameter assumptions made, all of which can be highly uncertain or incomplete, especially at the start of an outbreak. ${ }^{22}$ The situation may be even more challenging in LMIC settings, where data are more limited.

To date, only a few observational studies have investigated the impact of the pandemic on the use of health services in any international context, but especially in LMICs. ${ }^{23}$ In South Africa, one study found that lockdowns were associated with a large and significant decline in the use of health services among children but not adults. ${ }^{24}$ In Karachi, Pakistan, a lockdown introduced in late March was associated with a more than $50 \%$ decline in routine immunisations. ${ }^{25}$ In Nepal, institutional delivery rates decreased by more than half during a lockdown period. ${ }^{26}$ No studies, to our knowledge, have yet investigated the impact of the lifting of such lockdown policies.

In early 2020, the Democratic Republic of the Congo (DRC) was already dealing with many competing outbreaks, including large-scale outbreaks of EVD and measles. ${ }^{27}$ Given its large population, densely populated cities and weak health system, it was considered to be highly vulnerable to COVID-19. ${ }^{1}$ As of 22 March 2021, however, the country had confirmed only 27552 cases and 726 deaths. ${ }^{2}$

Policymakers need real-time data and evidence to inform decisions during outbreaks. ${ }^{28}$ Data from routine health information systems (RHIS) are now widely available in LMICs and have been used to conduct similar analyses during other infectious disease outbreaks. ${ }^{29} 1730$ In this study, we use RHIS data to evaluate the impact of COVID-19 and its related response measures on the use of health services in Kinshasa during the first wave of the pandemic (March to September) to provide insights to inform the ongoing response and future infectious disease outbreaks.

\section{METHODS \\ Context}

With a population of over 14 million, Kinshasa is one of the largest and most densely populated cities in Africa. The DRC health system is organised into health zones, which are further disaggregated into health areas. Each health zone should have at least one hospital, while each health area should have at least one health centre. Currently, Kinshasa has 851 health centres and 121 hospitals-some of which were designated COVID-19 treatment centres (figure 1). The city is subdivided into 24 communes, or municipalities, including Gombe, which is one of the more central and affluent communes. Gombe is a mixed residential and business district. It is also the home to many national and provincial government buildings as well as the Kinshasa Provincial Hospital. There is also an active private sector, which plays an important complementary role in delivering health services. ${ }^{31}$

The first case of COVID-19 in the DRC was identified on 10 March $2020 .^{32}$ The government immediately introduced an outbreak management and control plan including a series of public health measures aimed at reducing transmission of the virus including the closure of bars, restaurants and schools a few days later which was subsequently followed by a declaration of a state of emergency, closing of international borders and restricting travel in and out of Kinshasa on 24 March 2020. On 6 April 2020, the commune of Gombe, at the time known as the epicentre of the epidemic, was locked down, which closed stores and restricted all non-essential travel in and out of the commune and limited all movement within the commune to essential travel only. Health facilities and pharmacies remained 


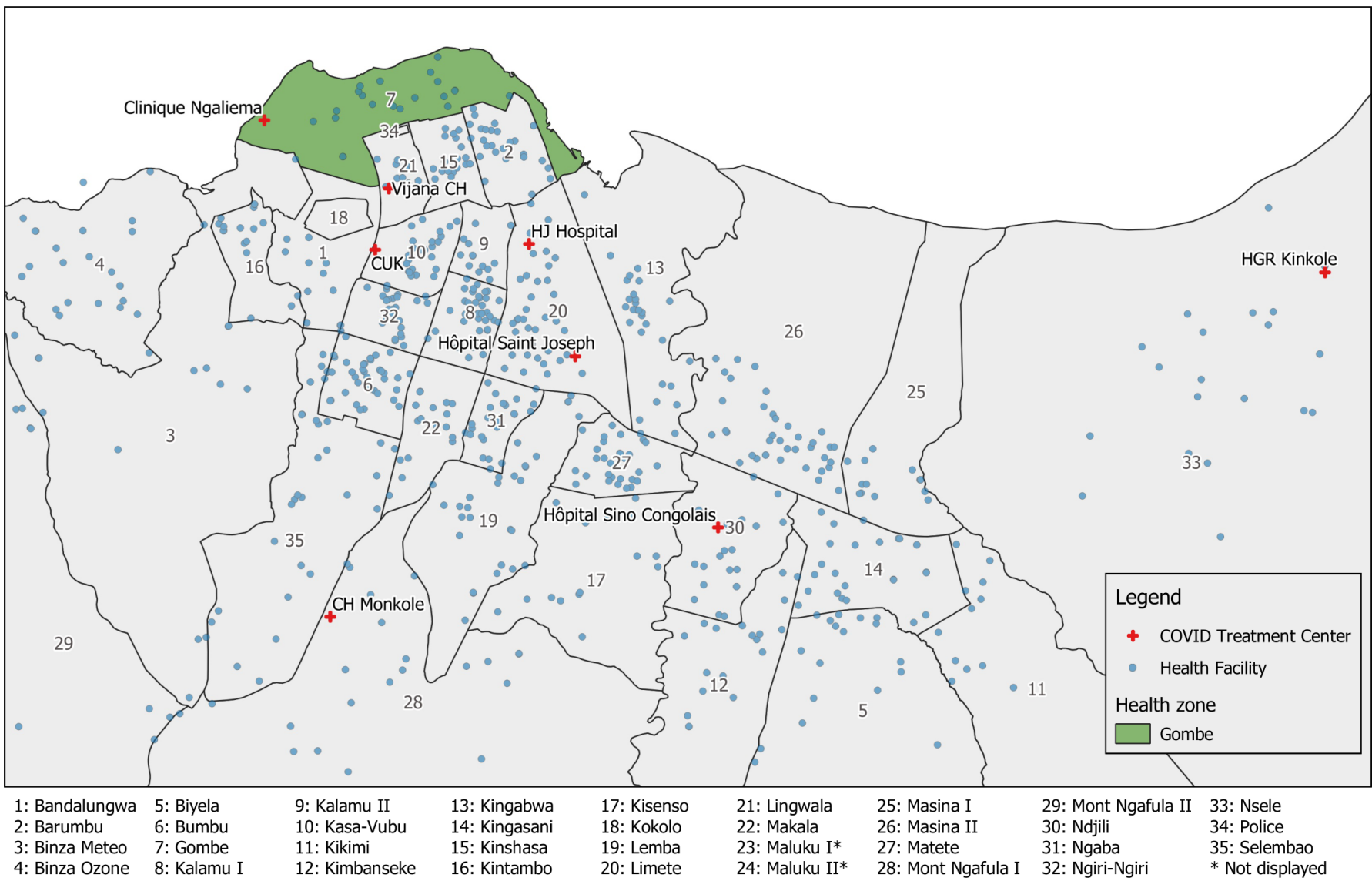

Figure 1 A map of Kinshasa with health zones outlined and showing eight health facilities, initially identified as centres for COVID-19 case treatment and hospitalisation (March to April 2020). The map only shows 33 health zones-two health zones (Maluku I and Maluku II) are not shown to optimise visibility. Gombe is highlighted in green.

open during this period and health-related travel was exempted from the lockdown (including for nonresidents who were still allowed to enter Gombe to access health services); however, there was no public transportation or taxis available within the commune. Free movement of transportation was allowed in other parts of Kinshasa. The lockdown was partially lifted on 22 April, allowing residents to purchase food and other essentials, but remained in place until 29 June. There

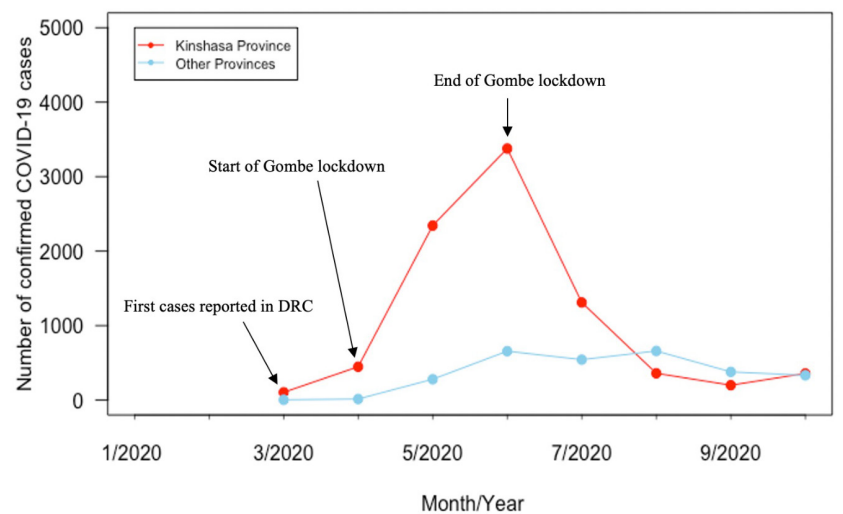

Figure 2 Monthly reported confirmed COVID-19 cases in Kinshasa and other Democratic Republic of the Congo (DRC) provinces. was no lockdown outside of the Gombe health zone. Figure 2 provides an overview of the confirmed cases of COVID-19 in Kinshasa and other DRC provinces.

\section{Data sources}

We used monthly time series data on service utilisation from the DRC Health Management Information System (HMIS), an electronic data collection system based on the District Health Information System 2 (DHIS2) platform. ${ }^{33}$ Specifically, we extracted data covering the pre-COVID-19 period (January 2018 to February 2020) and the COVID-19 period (March to December 2020). These data are input from health facilities' monthly health service use reports at district health offices. Considerable efforts have been made to improve the quality of HMIS data in DRC, including continual quality assessment activities at both the health zone and facility levels and incentives for report submission and completion. ${ }^{33}$ The data in this system have been used previously by the research team to conduct other evaluation projects. ${ }^{30}$ Data on COVID-19 cases were obtained from government sources and data on major policy responses were collected using official government sources. ${ }^{2}$

\section{Population}

Our unit of observation was the facility month. Our study sample included health facilities (ie, health centres and 
Table 1 Parameter estimates of the overall effect of COVID-19 on rates of total outpatient visits, visits for common infectious diseases and non-communicable diseases and maternal and child health services in Kinshasa, DRC

\begin{tabular}{|c|c|c|c|c|}
\hline \multirow[b]{2}{*}{ Indicators } & \multicolumn{2}{|l|}{ Level change } & \multicolumn{2}{|l|}{ Trend change } \\
\hline & IRR (95\% CI) & $P$ value & IRR (95\% CI) & $P$ value \\
\hline \multicolumn{5}{|c|}{ Overall visits and by sex, age and facility tier } \\
\hline Total outpatient visits & 0.75 (0.73 to 0.75$)$ & $<0.001$ & 1.01 (1.00 to 1.02$)$ & $<0.001$ \\
\hline Female & $0.76(0.73$ to 0.79$)$ & $<0.001$ & 1.01 (1.00 to 1.02$)$ & $<0.001$ \\
\hline Male & 0.74 (0.71 to 0.77$)$ & $<0.001$ & 1.01 (1.00 to 1.01$)$ & $<0.001$ \\
\hline Under 5 years & 0.75 (0.73 to 0.77$)$ & $<0.001$ & 1.00 (1.00 to 1.01$)$ & 0.01 \\
\hline 5 years and over & 0.75 (0.73 to 0.77$)$ & $<0.001$ & 1.01 (1.00 to 1.02$)$ & $<0.001$ \\
\hline Health centres & 0.86 (0.84 to 0.89$)$ & $<0.001$ & 1.01 (1.00 to 1.01$)$ & $<0.001$ \\
\hline Hospitals & 0.57 (0.53 to 0.62$)$ & $<0.001$ & $1.02(1.00$ to 1.03$)$ & 0.009 \\
\hline COVID-19 hospitals & $0.48(0.31$ to 0.74$)$ & $<0.001$ & $1.03(0.97$ to 1.11$)$ & 0.27 \\
\hline Non-COVID-19 hospitals & 0.58 (0.53 to 0.63$)$ & $<0.001$ & 1.01 (1.00 to 1.02$)$ & 0.02 \\
\hline \multicolumn{5}{|l|}{ Common IDs } \\
\hline Malaria case diagnoses & 0.76 (0.73 to 0.79$)$ & $<0.001$ & 1.01 (1.00 to 1.02$)$ & 0.001 \\
\hline RDT for malaria done & 0.70 (0.67 to 0.73$)$ & $<0.001$ & 1.01 (1.01 to 1.02$)$ & $<0.001$ \\
\hline Diarrhoea case diagnoses & 0.74 (0.70 to 0.78$)$ & $<0.001$ & 1.01 (1.00 to 1.02$)$ & $<0.001$ \\
\hline Pneumonia case diagnoses & 0.70 (0.65 to 0.74$)$ & $<0.001$ & 1.03 (1.02 to 1.04$)$ & $<0.001$ \\
\hline \multicolumn{5}{|l|}{ NCDs } \\
\hline Diabetes new case diagnoses & 0.61 (0.55 to 0.66$)$ & $<0.001$ & $1.03(1.01$ to 1.04$)$ & $<0.001$ \\
\hline Hypertension new case diagnoses & $0.84(0.79$ to 0.89$)$ & $<0.001$ & 1.00 (0.99 to 1.02$)$ & 0.15 \\
\hline \multicolumn{5}{|l|}{ Maternal health } \\
\hline ANC1 visits & 1.04 (1.01 to 1.07$)$ & 0.007 & 0.99 (0.99 to 1.00$)$ & 0.71 \\
\hline Facility-based childbirth & $1.08(1.05$ to 1.11$)$ & $<0.001$ & $1.00(0.99$ to 1.01$)$ & 0.32 \\
\hline PNC2 visits & 1.07 (1.03 to 1.11$)$ & $<0.001$ & 1.00 (0.99 to 1.01$)$ & 0.16 \\
\hline \multicolumn{5}{|l|}{ Vaccinations } \\
\hline DTP1 vaccine administered & 1.01 (0.98 to 1.04$)$ & 0.40 & 1.00 (0.99 to 1.01$)$ & 0.26 \\
\hline BCG vaccine administered & 0.95 (0.90 to 1.01$)$ & 0.08 & 1.01 (1.00 to 1.02$)$ & 0.001 \\
\hline OPV1 administered & 1.04 (1.01 to 1.08$)$ & 0.004 & 1.00 (1.00 to 1.01$)$ & 0.005 \\
\hline PCV-13 administered & 1.00 (0.97 to 1.04$)$ & 0.60 & $1.00(0.99$ to 1.01$)$ & 0.47 \\
\hline
\end{tabular}

Parameter estimates are from mixed effects segmented regression models comparing the period before COVID-19 (January 2018 to February 2020) and after the onset of COVID-19 (April to December 2020) in DRC.

ANC1, first antenatal care; DRC, Democratic Republic of the Congo; DTP, diphtheria-tetanus-pertussis; ID, infectious disease; IRR, incidence rate ratio; NCD, non-communicable disease; OPV, oral poliovirus vaccine; PCV, pneumococcal conjugate vaccine; PNC2, second postnatal care; RDT, rapid diagnostic test.

hospitals) across Kinshasa that reported consistently through DHIS2 during the study period. Because not all health facilities provide all health services, we used facility reporting patterns in the HMIS database for each service to determine whether a given health facility should be deemed a facility that provides a relevant service. Specifically, a facility had to have reported a service (eg, facilitybased childbirth) at least 1 month into the database to be considered as a facility that provides delivery care. Additionally, we included health facilities for each service that had a reporting rate of at least $25 \%$ both before COVID-19 and after the onset of the pandemic. Further, facilities with consecutive missing observations and/or outliers for a specific service were excluded from our sample. Because of these inclusion/exclusion criteria, the number of facilities included in our final analytical sample varied by indicator (see online supplemental table 1). Most health centres provide all services we studied; most hospitals provide all services except vaccinations that are not primarily provided at the hospital level (see online supplemental table 1). We excluded health posts, which provide largely health promotion and community health services, and private health facilities because their reporting rates are limited.

\section{Outcomes}

We evaluated the impact of COVID-19 on 14 indicators of health service utilisation: 

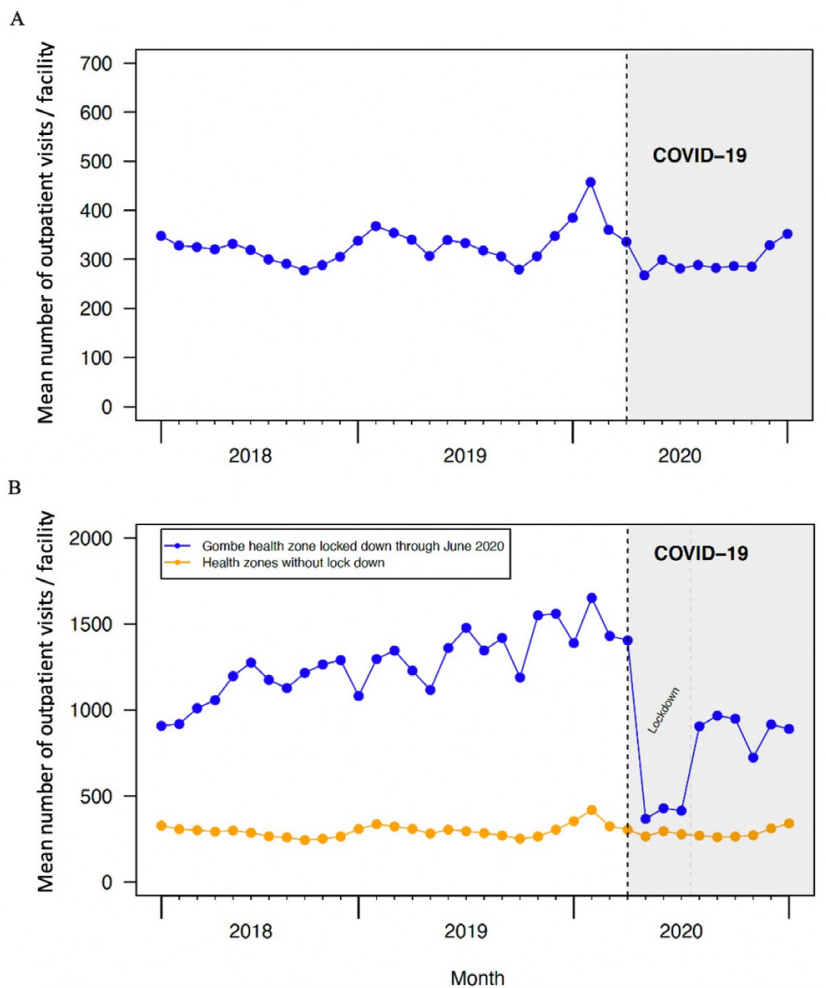

Figure 3 Time series of the mean number of total outpatient visits for (A) all of Kinshasa and (B) Gombe and other health zones without a lockdown, 2018-2020.

- Total outpatient visits: outpatient consultations, which include common infectious diseases and noncommunicable diseases (NCD) described below. It does not include visits for maternal health and vaccinations, which are tracked with other indicators described below.

- Common infectious diseases: uncomplicated pneumonia cases diagnosed; uncomplicated diarrhoea cases diagnosed; uncomplicated malaria cases diagnosed, and rapid diagnostic tests (RDT) for malaria conducted.

- Maternal health: first antenatal care (ANC1) visit, facility-based childbirth, second postnatal care (PNC2) visit within 6 days of birth.

- Vaccinations: first doses of diphtheria-tetanus-pertussis vaccine administered, BCG vaccine, oral poliovirus vaccine (OPV1) and the first dose of pneumococcal conjugate vaccine (PCV-13).

- NCDs: hypertension and diabetes cases newly diagnosed.

These indicators were selected because they accounted for the majority of primary care services provided by health facilities as well as those we believed could be influenced by the pandemic (see online supplemental table 2) as well as indicators with relatively high completeness reporting rates, except for the pneumonia and the NCD indicators, which had median reporting rates less than $60 \%$ but were still included to provide a more comprehensive picture of health service utilisation.

\section{Analytical strategy}

We used interrupted time series (ITS) analyses to assess the impact of the onset of the pandemic and the government response measures, using monthly time series data, while controlling for secular trends in the outcomes. ${ }^{34} 35$ As March 2020 was partially exposed to the pandemic and was also not exposed to the Gombe lockdown, we excluded it from our analyses by defining the start of both events as April 2020 and the Gombe lockdown period as April to June 2020. As baseline rates in health service volume vary across health facilities, we employed segmented quasi-Poisson mixed effects models, with health facility catchment population as an offset to estimate the impact on each indicator immediately following the start of the pandemic or the Gombe lockdown (level change) and over time (trend change) (see online statistical appendix). All our models were also adjusted for seasonality. Additionally, models for total outpatient visits and visits for common infectious diseases included a dummy variable to adjust for an unrelated pneumonia outbreak that took place in Kinshasa from December 2019 to February 2020. We also provide results from analyses that were not adjusted for the pneumonia outbreak in the online supplemental table 3 as a sensitivity analysis.

We defined outliers for each indicator as any observation exceeding seven SDs from the meantime trend estimated using facility-level local regression, which were subsequently treated as missing observations. Missing data were imputed using seasonally decomposed missing value imputation, accounting for seasonal patterns in the service utilisation time series data. ${ }^{36}$ We also performed sensitivity analyses using complete case analyses-that is, analyses that include facilities that had complete reporting or no missing values during the study period.

We run our ITS models on all health zones in Kinshasa to quantify the effect of the pandemic across the city (see Statistical Appendix). We also conducted subgroup analyses. First, wherever possible, we stratified our analyses by the Gombe versus the remaining 34 health zones to estimate the additional impact of the lockdown versus COVID-19 alone. For the Gombe health zone, we also ran models that included segments (level and trend changes) for the lockdown (April to June 2020) and postlockdown (July to December 2020) periods, allowing us to also estimate the impact of stopping the policy (see Statistical Appendix). Second, we conducted additional analyses to investigate whether the pandemic had a differential impact on different groups, specifically we stratified our sample by sex, age and health facility type wherever feasible. We report parameter estimates using the incidence rate ratio (IRR) and related 95\% CI. We also present changes visually using monthly time series indicating mean service utilisation per facility. All analyses were conducted using R V.4.0.2.

\section{Role of the funding source}

The funder of the study had no role in study design, data collection, data analysis, data interpretation or writing of 
the report. All authors had full access to all the data in the study and had final responsibility for the decision to submit for publication.

\section{Patient and public involvement}

This research was done without patient involvement. Patients were not invited to comment on the study design and were not consulted to develop patientrelevant outcomes or interpret the results. Patients were not invited to contribute to the writing or editing of this document for readability or accuracy.

\section{RESULTS}

Of 975 health facilities that reported through DHIS2 in Kinshasa from January 2018 to December 2020, thirty-one were in the Gombe health zone and 940 in other health zones (online supplemental table 1). The number of facilities that reported at least once for each service ranged from 760 for PCV-13 administered to 971 for total outpatient visits. Similarly, the number of facilities that was included in our final analytical sample varied by services, ranging from 435 (56.94\%) for OPV1 administered to $853(87.85 \%)$ for total outpatient visits. Overall, our analyses included 9158657 total outpatient visits including 3282890 visits for infectious diseases (malaria, diarrhoea and pneumonia) and 256405 new diagnoses of NCDs (diabetes and hypertension), and 3467713 maternal and child health services (ANC1 visits, facilitybased childbirth, PNC2 visits and vaccinations) between January 2018 and December 2020 (see online supplemental table 4).

\section{Total outpatient visits}

We found that the overall use of health service decreased following the start of the pandemic (table 1). Rates of total outpatient visits immediately decreased by $25 \%$ (IRR: $0.75,95 \%$ CI 0.73 to 0.75 ) relative to the prepandemic period (table 1, figure $3 \mathrm{~A}$ ). However, rates of total outpatient visits increased marginally after the initial decline, but significantly over time relative to the trend that would have been expected without COVID-19 (IRR: $1.01,95 \%$ CI 1.00 to 1.02 ). Results stratified by zones with and without lockdown showed that the policy resulted in an immediate decrease of over $80 \%$ in rates of all outpatient visits in Gombe (IRR: 0.19, 95\% CI 0.12 to 0.32 ); however, the rates of outpatient visits immediately increased by more than twofold when the lockdown was lifted (IRR: 2.50, 95\% CI 1.81 to 3.45 ), but remained lower than prepandemic rates by approximately $16 \%$ (table 2, figure $3 \mathrm{~B}$ and online supplemental table 6). In contrast, health zones without lockdown had relatively smaller declines of about only $15 \%$ (IRR: $0.84,95 \%$ CI 0.81 to 0.86 ) (table 2 and figure $3 \mathrm{~B}$ ).

Analyses stratified by sex showed that there was a similar immediate reduction in the outpatient visits in both female and male patients across Kinshasa (table 1 and online supplemental figure 1). Similarly, decreases in service use were very similar among under 5 -year patients compared with those over 5 years of age (table 1 ). However, immediate reductions in the total outpatient visits were substantially higher in hospitals (IRR: 0.57 , 95\% CI 0.53 to 0.62 ) than in health centres (IRR: 0.86, $95 \%$ CI 0.84 to 0.89 ) (table 1 and online supplemental figure 2). Additional analysis stratified by COVID-19 and non-COVID-19 hospitals showed that reductions in service use were even higher in COVID-19 hospitals (table 1).

\section{Common infectious diseases}

The start of the pandemic was associated with an immediate decrease in rates of visits for common infectious diseases, ranging from $24 \%$ reduction in visits for malaria (IRR: $0.76,95 \%$ CI 0.73 to 0.79 ) to $30 \%$ for pneumonia visits (IRR: $0.70,95 \%$ CI 0.65 to 0.74 ) (table 1). Important drops in RDTs were also observed. However, the trends of rates of visits for infectious diseases increased over time marginally, but significantly, following the start of the pandemic (table 1). Our stratified analyses showed that, overall, decreases in rates of visits for malaria, diarrhoea and pneumonia were substantially larger in Gombe relative to the other health zones (table 2 and figure 4). In particular, in Gombe, outpatient visits decreased immediately by $90 \%$ for malaria (IRR: $0.10,95 \%$ CI 0.05 to 0.20 ), $81 \%$ for diarrhoea (IRR: $0.19,95 \%$ CI 0.08 to 0.43 ) and $75 \%$ for pneumonia (IRR: $0.25,95 \%$ CI 0.09 to 0.69 ). However, the use of health services also immediately increased following the end of the lockdown by ranging from approximately $60 \%$ for pneumonia to $75 \%$ for diarrhoea and RDTs increased by more than twofold during the same time period (table 2 and figure 4 ). It should be noted that these increases remained lower than the prepandemic rate (online supplemental table 6 ). In the health zones without a lockdown, visits for these infectious diseases declined by about only 20\%-30\% immediately after onset of pandemic and stayed similar in the postlockdown pandemic period (table 2 and figure 4).

\section{Non-communicable diseases}

The pandemic was associated with an immediate decline in rates of visits for new diagnosis of NCDs: a $39 \%$ drop for diabetes (IRR: $0.61,95 \%$ CI 0.55 to 0.66 ) and $16 \%$ drop for hypertension (IRR: $0.84,95 \%$ CI 0.79 to 0.89 ) were observed (table 1). Stratified analysis showed that in Gombe, immediately following the COVID-19 and lockdown policy, visits decreased by 93\% (IRR: $0.07,95 \%$ CI 0.03 to 0.19 ) for new patients with diabetes and $77 \%$ for new patients with hypertension (IRR: 0.23 , 95\% CI 0.07 to 0.70 ); however, in the postlockdown pandemic period, visits immediately increased by more than $50 \%$ for both diabetes and hypertension, but they still remained about $35 \%$ lower than in the prepandemic period (see table 2, figure 5 and online supplemental table 6 ). In the health zones without a lockdown, visits declined by only $10 \%$ for 


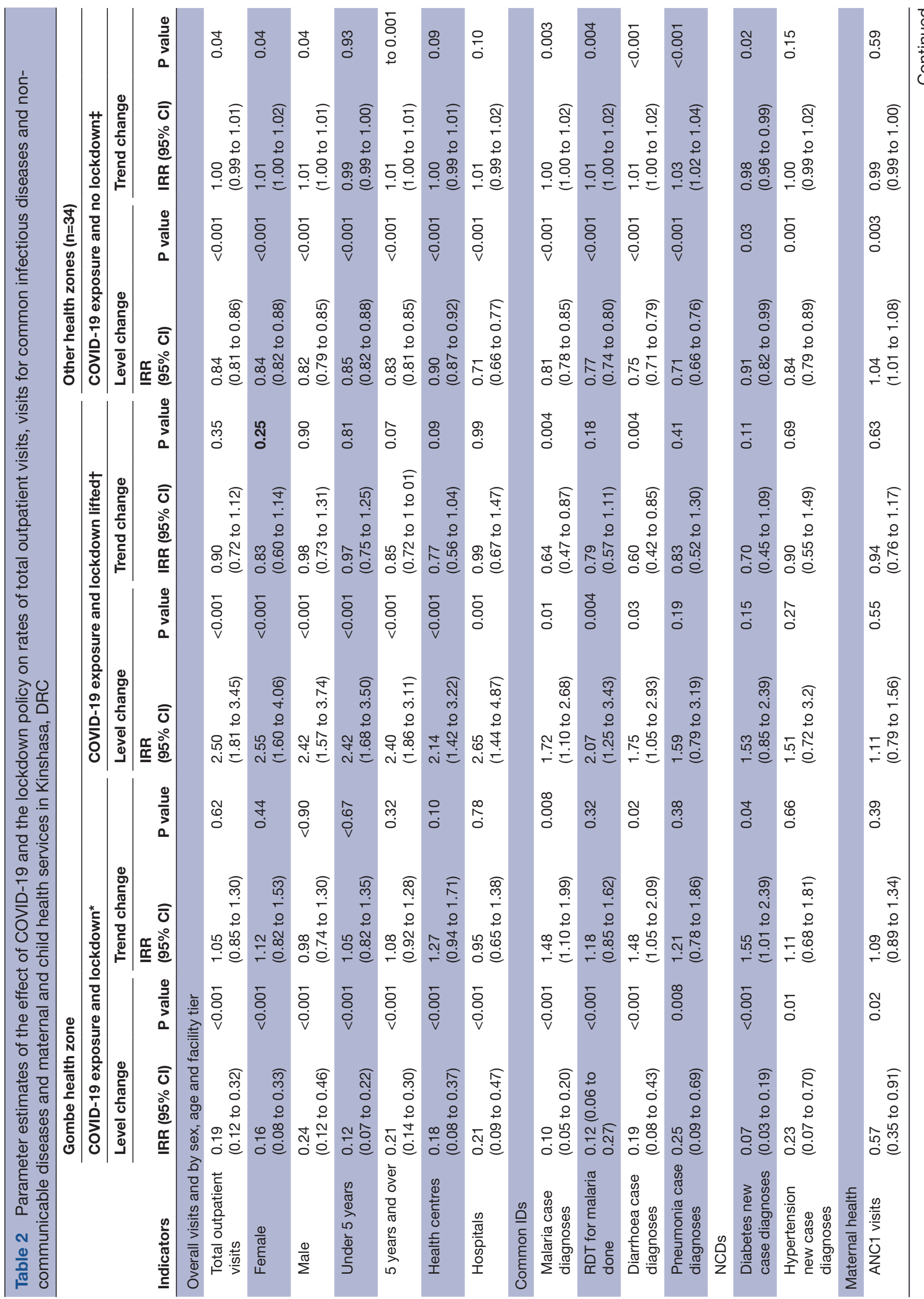

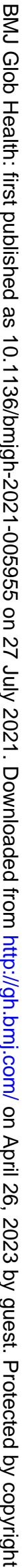




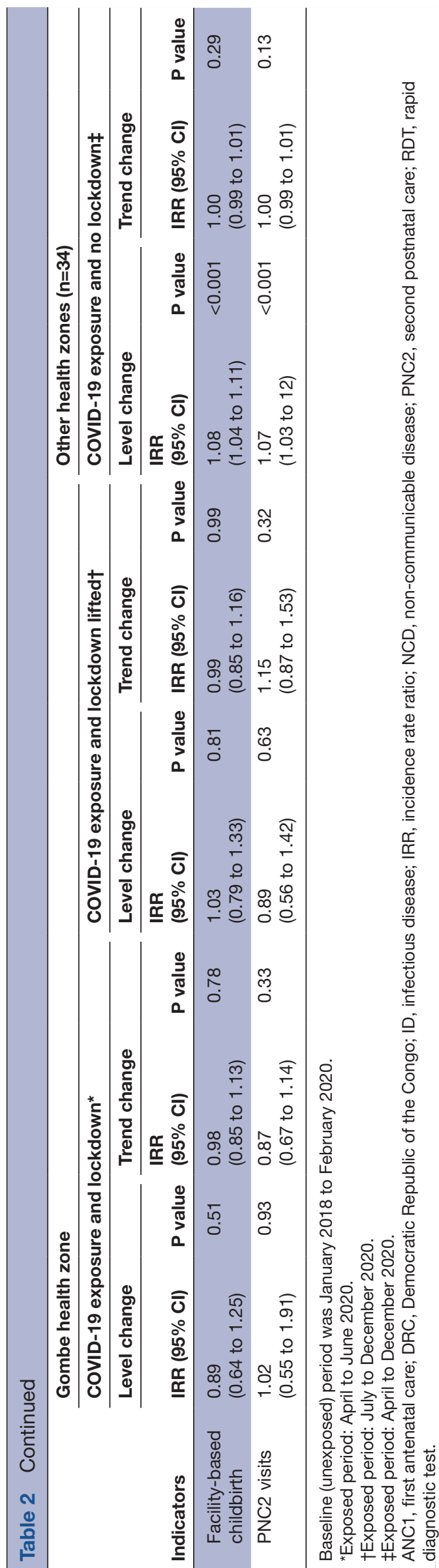

diabetes (IRR level change: $0.91,95 \%$ CI 0.82 to 0.99 ) and $15 \%$ for hypertension (IRR: $0.84,95 \%$ CI 0.79 to 0.89 ) (see table 2 and figure 5 ) and stayed similar in the postlockdown pandemic period (table 2 and figure 5).

\section{Maternal health}

Unlike other health services, rates of ANC1 visits, facilitybased childbirth and PNC2 visits increased modestly immediately following the start of pandemic (table 1). However, the trend in rates of maternal health services did not increase significantly over time as compared with the trends that would have been expected without COVID-19 (table 1). When examining changes in rates of the use of these maternal health services by lockdown policy, we found that overall rates of facility-based childbirth and visits for PNC2 were not substantially affected by the pandemic nor the lockdown policy (table 2, figure $6 \mathrm{~B}$ and online supplemental figure 3). However, the rate of visits for ANC decreased by $\sim 45 \%$ in Gombe (IRR: $0.57,95 \%$ CI 0.35 to 0.91 ) immediately following the start of the pandemic and lockdown policy (table 2 and figure 6A).

\section{Vaccinations}

Vaccinations were largely not affected by COVID-19 in Kinshasa (table 1). Given few facilities $(n=3$, see online supplemental table 1) in the Gombe health zone reported vaccinations consistently during the study period, we were not able to perform additional subgroup analyses to understand the effect of the lockdown policy on vaccinations in the Gombe health zone.

\section{Sensitivity analyses}

Findings from the complete case analyses were largely comparable to the reported results (see online supplemental table 5).

\section{DISCUSSION}

We found that the use of health services immediately decreased following the start of the COVID-19 pandemic in Kinshasa. Sizeable declines were observed for total outpatient visits, as well as for visits for common infectious diseases (pneumonia, diarrhoea, malaria) and for new diagnoses of NCDs (diabetes and hypertension). While substantial reductions were recorded in Gombe (eg, 83\% for total visits), reductions were much less pronounced in other areas of Kinshasa (eg, $\sim 16 \%$ for total visits) but still represented a substantial decline in the use of health services. We interpret this finding to suggest that while the pandemic, as well as government response to the pandemic, led to important declines in the use of health services, the lockdown policy had a much more important and more negative effect on the use of health services. Hospitals were more affected than health centres, a pattern that could potentially be explained if fear was a factor responsible for declines in health service use since patients might be more likely to avoid the types of facilities where patients with COVID-19 were most likely to be treated. Maternal health services and vaccinations, however, were considerably less affected which could be explained if 


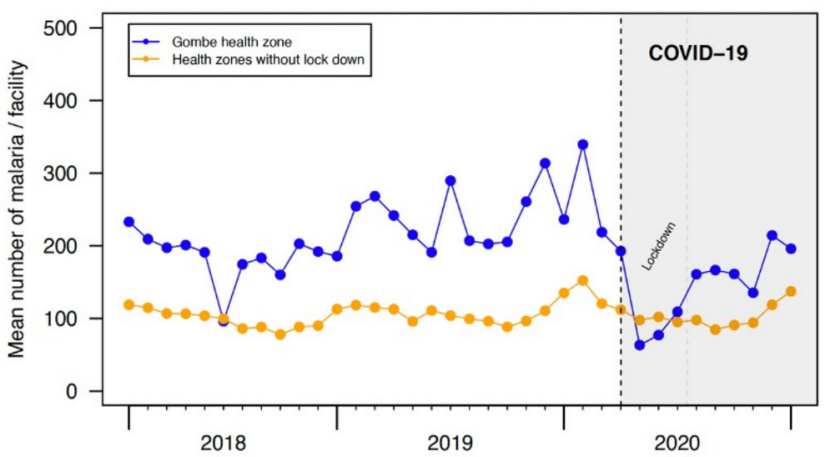

C

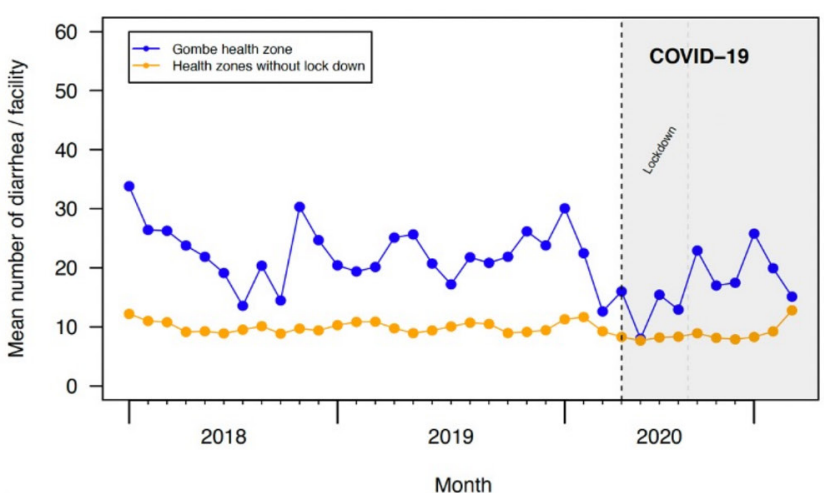

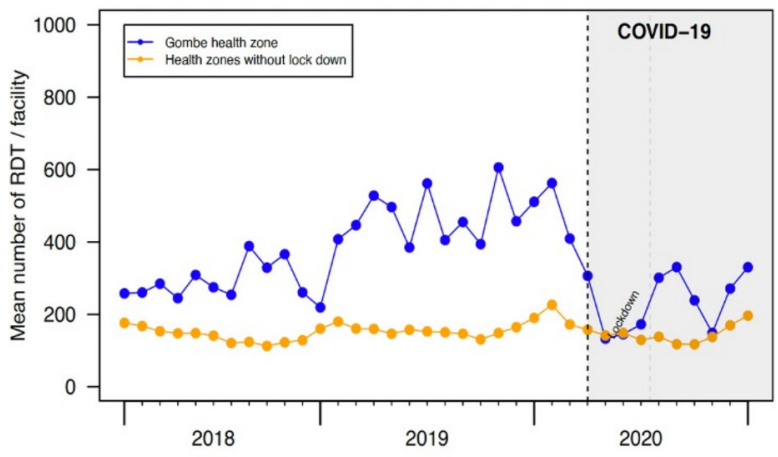

D

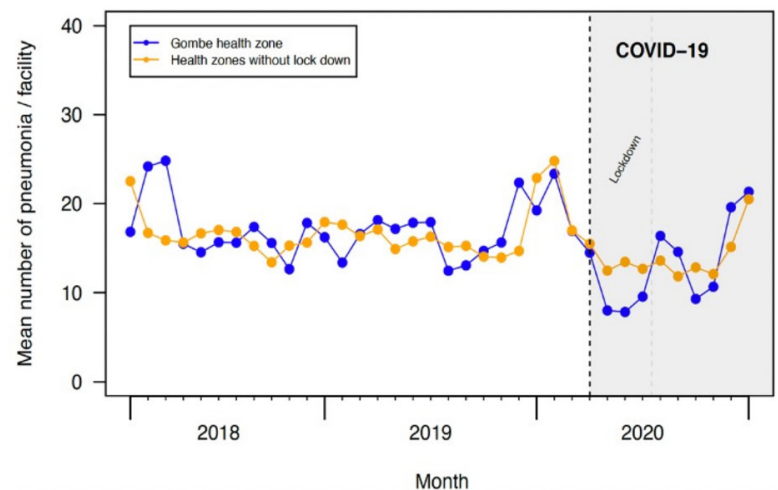

Figure 4 Time series of the mean number of $(A)$ malaria diagnoses, $(B)$ rapid diagnostic tests (RDT) for malaria, (C) diarrhoea diagnoses, and (D) pneumonia diagnoses, Gombe, in comparison to other health zones without a lockdown, Kinshasa, 20182020.

the women who would have otherwise travelled to Gombe, for example, at the provincial hospital, gave birth closer to home during lockdown. The fact that most maternal and child health services tend to be delivered at health centres rather than hospitals might also explain the smaller effects observed for these outcomes. Following the full termination of the lockdown policy, total outpatient visits, as well as visits for infectious diseases and NCDs, increased immediately by about twofold; however, this increase was not sustained over time and for most indicators utilisation rates did not return to prepandemic levels, suggesting this policy may have also had longer run effects. Although we found important reductions in the use of health services, we did not observe decreases as large as those predicted by modelling studies at the outset of the pandemic, except in Gombe.

Our study has several limitations. First, while we had a relatively long period of data before COVID-19, the periods during the lockdown included only three data points. While three data points are the minimum recommended for ITS studies, this rather short period may have affected the power of our study. Second, our analyses did not include all health facilities in Kinshasa. Specifically, we excluded health posts and other health facilities due to low reporting rates, as well as private health facilities. Similarly, several health centres and hospitals in Kinshasa were not included in our analyses because of inconsistent reporting of data. Because of these exclusions, our results should not be interpreted as population-level decreases in health service utilisation in Kinshasa. Third, given that only a small fraction of health facilities in the Gombe health zone reported vaccinations consistently during the study period, we were not able to perform additional subgroup analyses to understand the effect of the lockdown policy on vaccinations in the Gombe health zone. Finally, as previously mentioned, the Provincial Hospital of Kinshasa, the largest hospital in the city and that provides specialised services to the whole city, is located in Gombe. Hence, the lockdown of the Gombe area also affected the use of health services outside of Gombe, which we could not isolate from the effect of the lockdown. However, ITS does not require a comparison area, thus the results are still consistent for each geographical region.

While our study has shed light into changes in the use of health services in Kinshasa, we cannot directly attribute these changes to specific mechanisms. From a policy perspective, it is important to determine if the observed changes were due mainly to changes in the availability or accessibility of services as opposed to the demand for health services, including any impact factors such as fear or financial access. ${ }^{14}$ Although hospitals and clinics had remained open in Gombe, and although essential 


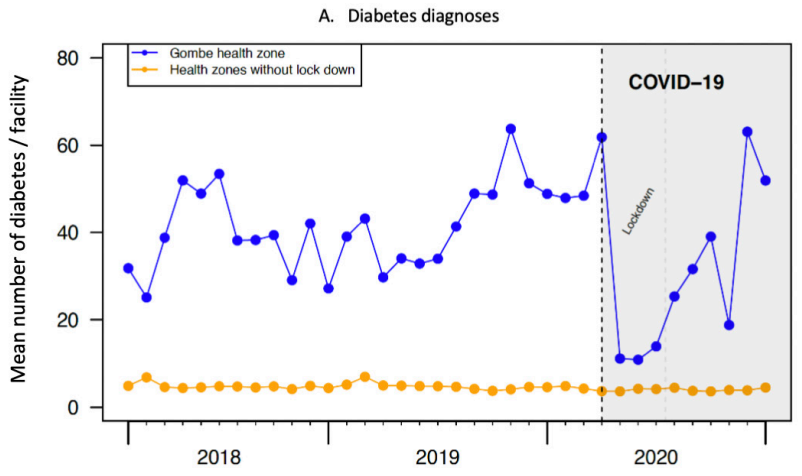

B. Hypertension diagnoses

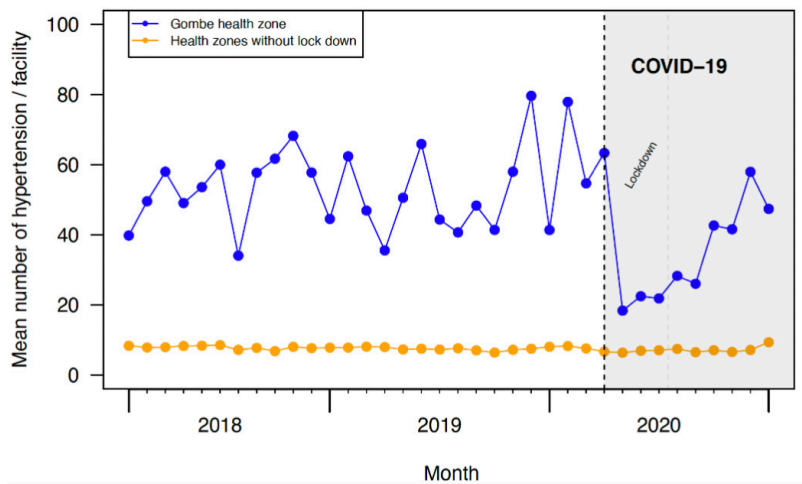

Figure 5 Time series of the mean number of new diagnoses of $(A)$ diabetes and $(B)$ hypertension, Gombe, in comparison to other health zones without a lockdown, Kinshasa, 20182020.

travel was still permitted, reductions in availability of transportation and the lack of clarity of the essentiality of some activities may have still limited people's ability to access services. However, the fact that hospitals, where COVID-19 treatment centres had been located, saw bigger declines than health centres provides some evidence that demand-side factors, such as fear, may have also played an important role. COVID-19 was a stigmatising disease in Kinshasa and as fever and respiratory symptoms are common reasons for consultations, people may have preferred to stay at home and practise self-medication rather than risk getting diagnosed as a case of COVID-19. Plus, early in the pandemic, in Kinshasa there was widespread awareness that comorbidities with NCDs could exacerbate COVID-19 outcomes, which could partially explain the very large drops observed for these health services.

Other factors, such as the decline in availability of RDTs for malaria due to international travel restrictions for several months, could also have played a role. Complementary qualitative studies evaluating the perception of the population vis-à-vis health service use during an epidemic as well as evaluating the economic impact on household level of a pandemic with concurrent lockdown measures are needed. Whether there was an important shift to the private sector is unknown and needs to be further assessed. However, there were no reports of overloaded private structures during this period.

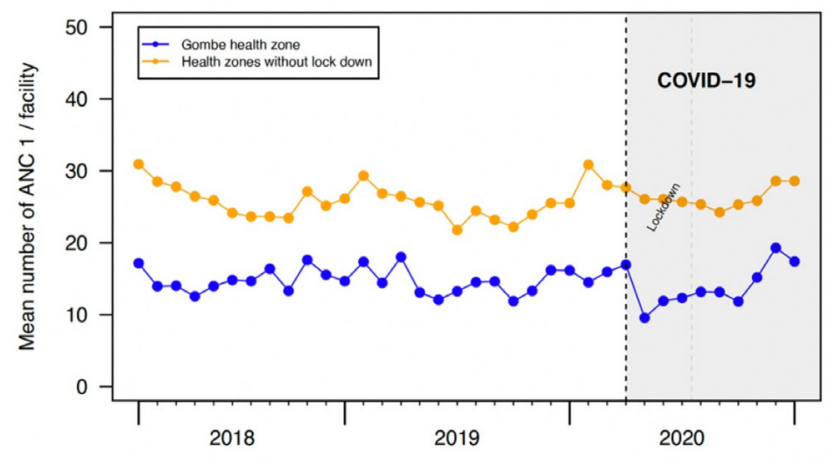

B

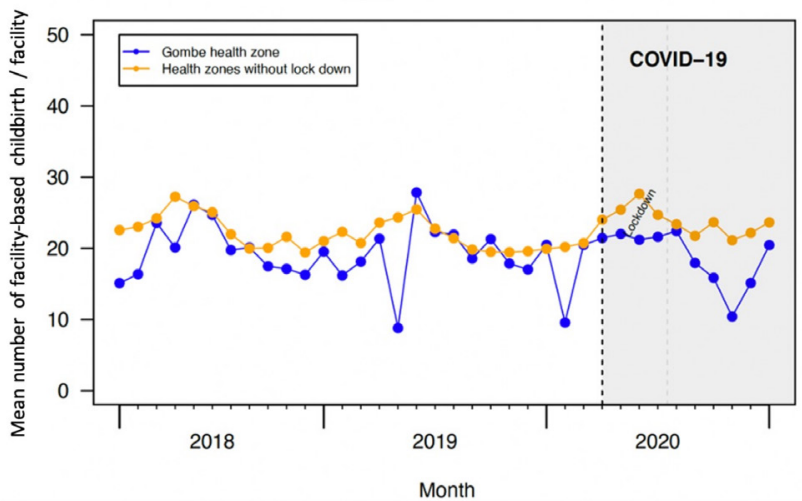

Figure 6 Time series of the mean number of $(A)$ visits for first antenatal care (ANC1) and (B) facility-based childbirth, Gombe, in comparison to other health zones without a lockdown, Kinshasa, 2018-2020.

The COVID-19 pandemic has led to substantial health, economic and social effects globally. In this study, we document important declines in the use of health services during the first wave of the pandemic in Kinshasa, mostly notably in the parts of the city that were subject to lockdown. A number of recent preprinted studies have also begun to document similar effects observed in other SSA countries, including in Sierra Leone ${ }^{37}$ where hospital services were observed to have declined, and in Kenya. ${ }^{38}$ More attention needs to be paid to ensuring the continuity of essential health services during outbreaks, especially during lockdown policies like the ones implemented in Gombe during the early phase of the pandemic. This could include providing more clear instructions on the essentiality of travel for health service utilisation and the provision of transportation for patients. Given the relatively low numbers of cases and deaths recorded in Kinshasa during this time, the effectiveness of such policies in containing COVID-19 needs to be weighed against its potential impact on population health. Integrating health information system data analysis with social sciences evidence can contribute to a comprehensive interpretation of the data. ${ }^{39}$

\section{Author affiliations}

${ }^{1}$ Department of Global Health and Population, Harvard TH Chan School of Public Health, Boston, Massachusetts, USA

${ }^{2}$ Public Health Emergencies, UNICEF, New York, New York, USA 
${ }^{3}$ Faculté de Médecine, Ecole de Santé Publique, Université de Lubumbashi, Lubumbashi, Democratic Republic of Congo

${ }^{4}$ Centre de Connaissances en Santé en RD Congo, Lubumbashi, Democratic Republic of Congo

${ }^{5}$ Ministère de la Santé Publique de la République Démocratique du Congo, Kinshasa, Democratic Republic of Congo

${ }^{6}$ Bluesquare, Brussels, Belgium

${ }^{7}$ Public Health School of Kinshasa, Université de Kinshasa, Kinshasa, Democratic Republic of Congo

${ }^{8}$ Ministry of Health of Democratic Republic of Congo, Kinshasa, Democratic Republic of Congo

${ }^{9}$ Institute of Tropical Medicine, Antwerp, Belgium

${ }^{10}$ School of Public Health, Li Ka Shing Faculty of Medicine, University of Hong Kong, Pokfulam, Hong Kong

Twitter Celestin Hategeka @drhategeka, Simone E Carter @simone_tweets, Grégoire Lurton @grlurton, Serge Ma-Nitu Mayaka @SergeMayaka and Karen Ann Grépin @KarenGrepin

Acknowledgements $\mathrm{CH}$ received support through a Banting Postdoctora Fellowship from the Canadian Institutes of Health Research.

Contributors $\mathrm{CH}$ designed the study, conducted the analysis and wrote the first draft of the article. SEC coordinated research activities and facilitated access to data. FMC, ENK, SMNM and DKM provided feedback on drafts of the article and provided data on policy response. EvK and VV contributed to the study design and drafting of the article. KAG conceptualised the research project, drafted the manuscript and provided overall oversight to the project.

Funding International Development Research Centre, Rapid Research Fund for Ebola Virus Disease Outbreak, Grant 108966-002 (Principal Investigator Karen Grépin).

Competing interests GL works for Bluesquare, which has ongoing contracts with a variety of organisations in DRC including the Ministry of Health and the World Bank.

\section{Patient consent for publication Not required.}

Ethics approval We used a research protocol that had been approved by the Ethics Committees at Wilfrid Laurier University (Canada) and Kinshasa School of Public Health (DRC). We also obtained authorisation from the Ministry of Public Health to use these data to evaluate the impact of the pandemic on health service utilisation.

Provenance and peer review Not commissioned; externally peer reviewed.

Data availability statement Data may be obtained from a third party and are not publicly available. Data can be obtained through the Ministry of Public Health in the DRC.

Supplemental material This content has been supplied by the author(s). It has not been vetted by BMJ Publishing Group Limited (BMJ) and may not have been peer-reviewed. Any opinions or recommendations discussed are solely those of the author(s) and are not endorsed by BMJ. BMJ disclaims all liability and responsibility arising from any reliance placed on the content. Where the content includes any translated material, BMJ does not warrant the accuracy and reliability of the translations (including but not limited to local regulations, clinical guidelines, terminology, drug names and drug dosages), and is not responsible for any error and/or omissions arising from translation and adaptation or otherwise.

Open access This is an open access article distributed in accordance with the Creative Commons Attribution Non Commercial (CC BY-NC 4.0) license, which permits others to distribute, remix, adapt, build upon this work non-commercially, and license their derivative works on different terms, provided the original work is properly cited, appropriate credit is given, any changes made indicated, and the use is non-commercial. See: http://creativecommons.org/licenses/by-nc/4.0/.

\section{ORCID iDs}

Celestin Hategeka http://orcid.org/0000-0001-7808-4652

Simone E Carter http://orcid.org/0000-0003-3818-3538

Faustin Mukalenge Chenge http://orcid.org/0000-0001-7679-2065

Grégoire Lurton http://orcid.org/0000-0002-3714-6893

Serge Ma-Nitu Mayaka http://orcid.org/0000-0002-9124-2114

Dieudonné Kazadi Mwamba http://orcid.org/0000-0002-4676-2495

Esther van Kleef http://orcid.org/0000-0002-3312-7185

Veerle Vanlerberghe http://orcid.org/0000-0002-6531-0793

Karen Ann Grépin http://orcid.org/0000-0003-4368-0045

\section{REFERENCES}

1 Cabore JW, Karamagi HC, Kipruto H, et al. The potential effects of widespread community transmission of SARS-CoV-2 infection in the world Health organization African region: a predictive model. BMJ Glob Health 2020;5:e002647.

2 WHO. Who coronavirus disease (COVID-19) Dashboard, 2021. Available: https://covid19.who.int/ [Accessed 11 Feb 2021].

3 Wilhelm JA, Helleringer S. Utilization of non-Ebola health care services during Ebola outbreaks: a systematic review and metaanalysis. J Glob Health 2019;9:010406.

4 Elston JWT, Cartwright C, Ndumbi P, et al. The health impact of the 2014-15 Ebola outbreak. Public Health 2017;143:60-70.

5 Helleringer S, Noymer A. Magnitude of Ebola relative to other causes of death in Liberia, Sierra Leone, and guinea. Lancet Glob Health 2015;3:e255-6.

6 Walker PGT, White MT, Griffin JT, et al. Malaria morbidity and mortality in Ebola-affected countries caused by decreased healthcare capacity, and the potential effect of mitigation strategies: a modelling analysis. Lancet Infect Dis 2015;15:825-32.

7 Sochas L, Channon AA, Nam S. Counting indirect crisis-related deaths in the context of a low-resilience health system: the case of maternal and neonatal health during the Ebola epidemic in Sierra Leone. Health Policy Plan 2017;32:iii32-9.

8 Takahashi S, Metcalf CJE, Ferrari MJ, et al. Reduced vaccination and the risk of measles and other childhood infections post-Ebola Science 2015;347:1240-2.

9 Plucinski MM, Guilavogui T, Sidikiba S, et al. Effect of the Ebolavirus-disease epidemic on malaria case management in guinea 2014: a cross-sectional survey of health facilities. Lancet Infect Dis 2015;15:1017-23.

10 Tattevin P, Baysah MK, Raguin G, et al. Retention in care for HIV-infected patients in the eye of the Ebola storm: lessons from Monrovia, Liberia. AIDS 2015;29:N1-2.

11 Leuenberger D, Hebelamou J, Strahm S, et al. Impact of the Ebola epidemic on general and HIV care in Macenta, forest guinea, 2014. AIDS 2015;29:1883-7.

12 Evans DK, Goldstein M, Popova A. Health-Care worker mortality and the legacy of the Ebola epidemic. Lancet Glob Health 2015;3:e439-40.

13 McLean KE, Abramowitz SA, Ball JD, et al. Community-Based reports of morbidity, mortality, and health-seeking behaviours in four Monrovia communities during the West African Ebola epidemic. Glob Public Health 2018;13:528-44.

14 Morse B, Grépin KA, Blair RA, et al. Patterns of demand for non-Ebola health services during and after the Ebola outbreak: panel survey evidence from Monrovia, Liberia. BMJ Glob Health 2016;1:e000007.

15 Delamou A, Ayadi AME, Sidibe S, et al. Effect of Ebola virus disease on maternal and child health services in guinea: a retrospective observational cohort study. Lancet Glob Health 2017:5:e448-57.

16 Camara BS, Delamou A, Diro E, et al. Effect of the 2014/2015 Ebola outbreak on reproductive health services in a rural district of guinea: an ecological study. Trans $R$ Soc Trop Med Hyg 2017;111:22-9.

17 Wagenaar BH, Augusto O, Beste J, et al. The 2014-2015 Ebola virus disease outbreak and primary healthcare delivery in Liberia: timeseries analyses for 2010-2016. PLoS Med 2018;15:e1002508.

18 Roberton T, Carter ED, Chou VB, et al. Early estimates of the indirect effects of the COVID-19 pandemic on maternal and child mortality in low-income and middle-income countries: a modelling study. Lancet Glob Health 2020;8:e901-8.

19 Hogan AB, Jewell BL, Sherrard-Smith E, et al. Potential impact of the COVID-19 pandemic on HIV, tuberculosis, and malaria in lowincome and middle-income countries: a modelling study. Lancet Glob Health 2020;8:e1132-41.

$20 \mathrm{WHO}$. The potential impact of health service disruptions on the burden of malaria, 2020. Available: https://www.who.int/ publications/i/item/9789240004641

21 Sherrard-Smith E, Hogan AB, Hamlet A, et al. The potential public health consequences of COVID-19 on malaria in Africa. Nat Med 2020;26:1411-6.

22 Press WH, Levin RC, Modeling LRC. Modeling, post COVID-19. Science 2020;370:1015.

23 Moynihan R, Sanders S, Michaleff ZA, et al. Impact of COVID-19 pandemic on utilisation of healthcare services: a systematic review. BMJ Open 2021;11:e045343.

24 Siedner MJ, Kraemer JD, Meyer MJ, et al. Access to primary healthcare during lockdown measures for COVID-19 in rural South Africa: an interrupted time series analysis. BMJ Open 2020;10:e043763. 
25 Chandir S, Siddiqi DA, Setayesh H, et al. Impact of COVID-19 lockdown on routine immunisation in Karachi, Pakistan. Lancet Glob Health 2020;8:e1118-20.

26 Kc A, Gurung R, Kinney MV, et al. Effect of the COVID-19 pandemic response on intrapartum care, stillbirth, and neonatal mortality outcomes in Nepal: a prospective observational study. Lancet Glob Health 2020;8:e1273-81.

27 Nachega JB, Mbala-Kingebeni P, Otshudiema J, et al. The colliding epidemics of COVID-19, Ebola, and measles in the Democratic Republic of the Congo. Lancet Glob Health 2020;8:e991-2.

28 Lal A, Ashworth HC, Dada S, et al. Optimizing pandemic preparedness and response through health information systems: lessons learned from Ebola to COVID-19. Disaster Med Public Health Prep 2020:1-8.

29 Hung YW, Hoxha K, Irwin BR, et al. Using routine health information data for research in low- and middle-income countries: a systematic review. BMC Health Serv Res 2020;20:790.

30 Hung YW, Law MR, Cheng L, et al. Impact of a free care policy on the utilisation of health services during an Ebola outbreak in the Democratic Republic of Congo: an interrupted time-series analysis. BMJ Glob Health 2020;5:e002119.

31 Bruner B, Combet V, Callahan S. The role of the private sector in improving the performance of the health system in the Democratic Republic of Congo. Bethesda, MD 2018.

32 AFRO W. First case of COVID-19 confirmed in the Democratic Republic of the Congo, 2020. Available: https://www.afro.who.int/ news/first-case-covid-19-confirmed-democratic-republic-congo [Accessed 11 Feb 2021].

33 Evaluation M. Mapping the Stages of MEASURE Evaluation's Data Use Continuum to DHIS 2: An Example from the Democratic Republic of the Congo. Chapel Hill, NC 2019.

34 Wagner AK, Soumerai SB, Zhang F, et al. Segmented regression analysis of interrupted time series studies in medication use research. J Clin Pharm Ther 2002;27:299-309.

35 Hategeka C, Ruton $\mathrm{H}$, Karamouzian M, et al. Use of interrupted time series methods in the evaluation of health system quality improvement interventions: a methodological systematic review. BMJ Glob Health 2020;5:e003567.

36 Feng S, Hategeka C, Grépin KA. Addressing missing values in routine health information system data: an evaluation of imputation methods using data from the Democratic Republic of the Congo during the COVID-19 pandemic 2021

37 Sevalie S, Youkee D, van DAJ, et al. The impact of the COVID-19 pandemic on health service utilisation in Sierra Leone. Medrxiv 2021.

38 Barasa E, Kazungu J, Orangi S. Assessing the indirect health impacts of the COVID-19 pandemic in Kenya 2021;1.

39 Carter SE, Gobat N, Pfaffmann Zambruni J, et al. What questions we should be asking about COVID-19 in humanitarian settings: perspectives from the social sciences analysis cell in the Democratic Republic of the Congo. BMJ Glob Health 2020;5:e003607. 